\title{
Alginate Capsules with Cuttlebone-derived Fillers as an Integrated Solution for Bone Repair
}

\author{
Alisa PALAVENIENE *, Migle LEBEDEVAITE, Jolanta LIESIENE \\ Department of Polymer Chemistry and Technology, Kaunas University of Technology, Radvilenu pl. 19, LT-50254 Kaunas, \\ Lithuania \\ crossref http://dx.doi.org/10.5755/j01.ms.24.3.18858
}

Received 20 August 2017; accepted 13 November 2017

\begin{abstract}
Alginate capsules with cuttlebone-derived fillers were developed for bone repair applications. Prepared capsules were designed to be suitable for the treatment of small-sized bone loss provocative diseases, such as endodontic and periodontal diseases. Cuttlebone microparticles, as a source of calcium carbonate, or cuttlebone-derived hydroxyapatite microparticles were used as mineral fillers for the preparation of alginate capsules. The capsules were additionally covered with chitosan layer for the hard structure formation and improvement of adhesive properties. Encapsulation efficiency of dexamethasone as a model allopathic drug was $19 \%, 24 \%$ and $12 \%$ for capsules with cuttlebone microparticles, capsules with cuttlebonederived hydroxyapatite and capsules without any mineral filler (control group), respectively. We observed that chitosan coating and lyophilisation contributed for the preservation of spherical structure of alginate capsules with mineral fillers. Swelling study of dried capsules in simulated physiological environment (in phosphate buffer saline, $\mathrm{pH}=7.2$ at $37^{\circ} \mathrm{C}$ temperature) showed that average swelling index of alginate-based capsules without mineral fillers was much higher in comparison to capsules with cuttlebone fillers: $121 \%, 17 \%$ and $3 \%$ for control group, capsules with cuttlebone microparticles and capsules with cuttlebone-derived hydroxyapatite, respectively.

Keywords: cuttlebone, hydroxyapatite, alginate capsules, glucono-delta-lactone, bone repair.
\end{abstract}

\section{INTRODUCTION}

Nowadays the use of marine-derived natural materials, such as cuttlebone (CB), sodium alginate, chitosan, etc., in biomedical engineering gains an enormous scale due to their high biocompatibility, biodegradability, non-toxicity, low immunogenicity, attractive cost and wide accessibility all over the world $[1,2]$. On that ground, great efforts are made towards the improvement of allopathic drugs delivery system in the treatment of bone loss provocative diseases, such as endodontic and periodontal diseases. Encapsulation of bioactive materials is employed as an effective and promising technique alongside the use of traditional drug delivery systems, such as injectable gel or films, strips, sponges, compacts and granules [3,4]. Abovementioned materials, for example, calcium phosphate granules, could be difficult to handle and to ensure sufficient contact with bone for further osteoconduction [5]. The fundamental of endodontic and periodontal diseases treatment is based on the elimination of inflammation and bacterial infection, while untreated periodontal pockets lead to the loss of teeth [6]. Regeneration of bone around the affected teeth is usually implemented by additional costly and protracted bone restoring procedures, i.e. bone grafting. The size of periodontal intrabone defects could vary depending on a stage of disease and other factors. However, the surgical intervention and treatment using graft materials is reasonable when the size of intrabony defects is $\geq 2 \mathrm{~mm}$ and probing depth $\geq 6 \mathrm{~mm}$ [7].

Cuttlebone (CB) is an endoskeleton of a marine mollusc Sepia officinalis. $\mathrm{CB}$ has an attractive perspective in biomedical applications, such as bone repair, due to its biocompatibility and osteoconductivity. The composition of $\mathrm{CB}$ includes $90 \%$ of inorganic ( $\mathrm{Ca}, \mathrm{Mg}, \mathrm{Na}, \mathrm{Fe}, \mathrm{Zn}, \mathrm{Sr}$, etc) and $10 \%$ of organic components, where calcium carbonate is the main inorganic compound. Elemental composition of $\mathrm{CB}$ is very similar to that of human bone tissue. Moreover, hydroxyapatite (HAp) synthesis from CB could implement towards the improvement of osteoconductive properties of the material $[8,9]$. Nevertheless, both calcium carbonate and HAp are established as suitable and effective alloplastic graft materials in periodontal therapy $[10,11]$.

Alginate salts are accepted as an effective material in drug delivery systems for alginate/ceramic composites in bone tissue engineering and other biomedical applications. In biological environment $\mathrm{COO}^{-}$functional groups of alginate interact with mucous glycoproteins for the improvement of mucoadhesive properties and sufficient delivery of therapeutic agents to the mucous [12]. Tønnesen and Karlsen [13] have described the potential of alginate in drug delivery systems, including the development of controlled-release or diffusion systems for local and/or systemic administration of biomolecules and allopathic drugs. Tetracycline-loaded chitosan microspheres were prepared by ionotropic gelation and various physicalchemical parameters, affecting drug entrapment, particle size and morphology, were investigated [14]. Many studies describing encapsulation of antibiotics into poly(lactic-coglycolic acid)-based matrices have been performed [15 - 17]. However, only small number of studies describing encapsulation of bone restoring minerals into polymer matrix have been published. Ferraz et al. [18] have

\footnotetext{
* Corresponding author. Tel.: +370-645-00270.

E-mail address: alisa.palavenis@gmail.com (A. Palaveniene)
} 
developed alginate/HAp microspheres loaded with antibiotics for treatment of periodontitis. Microspheres had a sustained drug release profile and demonstrated osteoblast proliferation. Stanislavov et al. [19] have prepared alginate/HAp beads for bone tissue engineering and evaluated some physical-chemical characteristics.

In this study, CB microparticles and CB-HAp were used as mineral fillers for the preparation of alginate capsules containing dexamethasone for bone repair applications, mainly for the treatment of small-sized bone loss provocative diseases, such as periodontal and endodontic diseases.

\section{MATERIALS AND METHODS}

Dexamethasone-loaded alginate solution was prepared by initial swelling of sodium alginate in distilled water for $2 \mathrm{~h}$ to obtain $2 \%$ sodium alginate solution. Further, dexamethasone sodium phosphate (DSP) was added to obtain $0.4 \%$ concentration of DSP in the prepared sodium alginate solution. Powdered CB was prepared by crushing and milling the $\mathrm{CB}$ pieces in an agar mortar and sieving with sieves to obtain $35-42 \mu \mathrm{m}$ fraction. $\mathrm{CB} / \mathrm{HAp}$ was synthesised from $\mathrm{CB}$ microparticles as described elsewhere [20]. Different CB-derived materials were then mixed with DSP/alginate solutions and three groups of suspensions were prepared. Suspensions were extruded slowly into a $\mathrm{CaCl}_{2}$ solution (containing glucono-delta-lactone (GDL) and chitosan mixture) through syringe with a needle. The materials used for a preparation of capsules are presented in Table 1.

Further, capsules were gently agitated using magnetic stirrer (IKA RET basic) for $2 \mathrm{~h}$ in an abovementioned crosslinking solution for hardening of capsule core, then washed with distilled water and lyophilised to obtain alginate-based beads. The term beads refer to the capsules that were lyophilised after a preparation.

For the evaluation of DSP encapsulation efficiency, phosphate buffered saline (PBS) solution was prepared by mixing appropriate amounts of $0.1 \mathrm{M} \mathrm{Na} \mathrm{HPO}_{4} \cdot 2 \mathrm{H}_{2} \mathrm{O}$ and $0.1 \mathrm{M} \mathrm{NaH} \mathrm{PO}_{4} \cdot 2 \mathrm{H}_{2} \mathrm{O}$ to reach $\mathrm{pH}=7.2$. DSP content was determined by dissolving $100 \mathrm{mg}$ of dry capsules in PBS $(\mathrm{pH}=7.2)$ under sonication for $15 \mathrm{~min}$ until complete dissolution, followed by centrifugation at 2,500 rpm for $10 \mathrm{~min}$. The samples were then filtered through $25 \mathrm{~mm}$ syringe filter with $\mathrm{GxF} / 0.45 \mu \mathrm{m}$ membrane and the filtrate was analysed spectrophotometrically at $240 \mathrm{~nm}$ against phosphate buffer $(\mathrm{pH}=7.2)$ as a blank (spectrophotometer Varian Cary 50 UV-VIS). The results were expressed as an amount of $0.4 \%$ DSP per $100 \mathrm{mg}$ of dry capsules. Drug concentration of tested solutions was calculated using the DSP standard curve. Correlation coefficient of the calibration curve was close to $1\left(\mathrm{R}^{2}=0.9969\right)$ and therefore was reliable for evaluation of DSP concentrations in unknown samples.
FT-IR spectroscopy analysis was performed using a FTIR spectrometer (Perkin-Elmer, Inc., Waltham, USA). The samples for FTIR analysis were prepared by mixing the finely powdered dried samples with potassium bromide in an agate mortar at a 1:50 ratio and pressing the mixture into homogeneous pellets. Mid-infrared spectral range data, corresponding to $4000-400 \mathrm{~cm}^{-1}$, was used; the resolution was $4 \mathrm{~cm}^{-1}$.

Mean particle size $(n=20)$ of dry samples of each formulation was determined using SEM technique (FEI Quanta 200 FEG, Oregon, USA) with an accuracy of $\pm 0.05 \mathrm{~mm}$. The structure and surface morphology were also analysed; lyophilised samples were attached to aluminium stubs with double side adhesive strip and examined.

The swelling studies were performed using dry capsules as described elsewhere [21] with some modifications. Swelling study was carried out in PBS at $\mathrm{pH}=7.2$. Accurately weighed amount of beads (approx. $30-45 \mathrm{mg}$ ) were soaked in PBS, placing them in a thermostat at $37^{\circ} \mathrm{C}$ temperature. The beads were put off from the solution at fixed time points, gently wiped with paper napkin and weighed. Swelling index $(S I)$ as a measure of weight change was calculated according the formula:

$S I(\%)=\frac{\left(\mathrm{w}_{1}-\mathrm{w}_{0}\right)}{\mathrm{w}_{0}} \times 100$,

where $W_{0}$ is an initial weight of beads and $W_{l}$ is a weight of beads in the swollen state at a given time point.

Data was presented as the mean value of three measurements \pm standard error of deviation (SED).

\section{RESULTS AND DISCUSSION}

Dexamethasone-loaded capsules with CB-derived fillers were prepared by ionic cross-linking method. Calcium chloride solution was used as a source of $\mathrm{Ca}^{2+}$ ions for alginate/calcium complexes formation in an acidic solution (Fig. 1). Moreover, calcium carbonate in CB composition was used as a supporting ionic crosslinking agent for slow and controlled gelation of alginate hydrogel [22], while GDL was used as a slowly hydrolysing molecule, inducing gradual fall in $\mathrm{pH}$ from neutral to acidic [23]. The addition of GDL affected gradual dissociation of $\mathrm{CaCO}_{3}$ to produce $\mathrm{Ca}^{2+}$ ions. The internal gelation resulting in a homogeneous distribution of alginate within the hydrogel was therefore achieved due to: a-addition of $\mathrm{CB}$ microparticles as a source of calcium carbonate into alginate solution and $b$-addition of GDL into continuous phase.

Three types of dexamethasone-loaded alginate capsules were prepared: a-with $\mathrm{CB}$ microparticles (DSP/CB); $\mathrm{b}$-with CB-HAp (DSP/CB-HAp) and $\mathrm{c}$-without any mineral filler (DSP/0). Capsules were dried using lyophilisation for the improvement of their storage and handling.

Table 1. The materials used for a preparation of dexamethasone-loaded capsules

\begin{tabular}{|c|c|c|c|c|}
\hline Type of capsules & Allopathic drug & Mineral filler & Core component & Cross-linking solution \\
\hline DSP/CB & \multirow{3}{*}{$0.4 \% \mathrm{DSP}$} & $10 \%$ powdered CB & \multirow{2}{*}{$2 \%$ sodium alginate } & $\begin{array}{c}1 \% \mathrm{CaCl}+5 \% \mathrm{GDL}+ \\
1.5 \% \mathrm{chitosan}\end{array}$ \\
& & $10 \% \mathrm{CB} / \mathrm{HAp}$ & & \\
& & - & & \\
\hline DSP/CB-HAp & & &
\end{tabular}




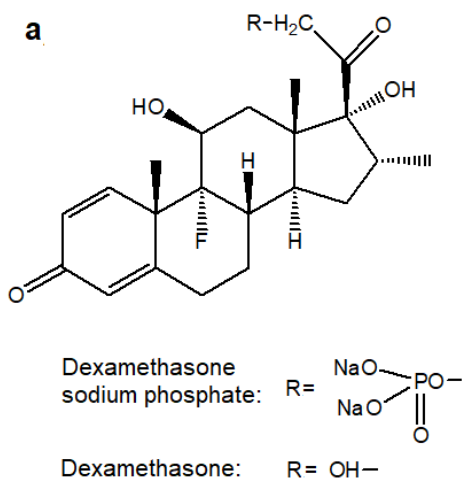

b

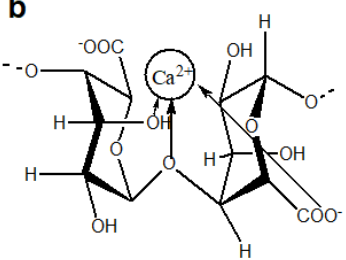

C

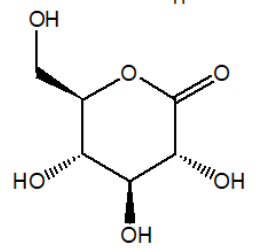

Fig. 1. Compositional elements of dexamethasone-loaded alginate matrix: a-dexamethasone and DSP structure; $\mathrm{b}$-alginate/calcium ion complex; $\mathrm{c}-\mathrm{GDL}$

In literature [24], the degradation of calcium carbonate or calcium phosphate in a composition of bone scaffold is assumed as positive phenomenon for successful bone regrowth, and the strategies to accelerate this process are thoroughly investigated. Sariibrahimoglu [24] studied the strategies to accelerate the degradation of calcium phosphates for bone tissue engineering. According to the study, the addition of GDL into polymer/mineral bone composites accelerates the degradation rate of poorly degradable apatitic calcium phosphate cements. Moreover, the solubility of carbonated apatite (like HAp derived from natural sources) generally increases because $\mathrm{CO}_{3}{ }^{2-}$ ions in the apatite lattice disturb $\mathrm{PO}_{4}{ }^{3-}$ and/or $\mathrm{OH}^{-}$ions from their lattice positions [25]. Following the latest achievements, in this study GDL and CB-HAp were added into polymer/mineral matrix for the improvement of bone repair related phenomena.

Dexamethasone (a synthetic glucocorticoid) was chosen as a model drug with the $\mathrm{C}=\mathrm{O}, \mathrm{COOH}$ and $\mathrm{P}-\mathrm{O}-\mathrm{C}$ functional groups. It is an immunomodulatory therapeutic agent, because the mechanism of action involves pleiotropic antiinflammatoric effects [26]. Reduction of inflammation is a most often emergent question in treatment of endodontic and periodontal diseases. Interestingly, immunomodulatory effect could play a key role in prevention of secondary bacterial infection [27] that is quite common after surgical intervention. Chitosan was added into continuous phase during ionic cross-linking process, because covering with chitosan layer contributes to the hard structure formation of capsules [28]. Prolonged stirring of capsules in calcium chloride solution during their preparation contributes to the dense cross-linked network formation, because deeper penetration of $\mathrm{Ca}^{2+}$ ions into alginate matrix could be achieved [29]. Moreover, coating with chitosan layer improves adhesive properties of alginate capsules significantly [13].

Encapsulation efficiency was calculated by means of drug loading into $100 \mathrm{mg}$ of dry capsules (Table 2).

Table 2. Encapsulation efficiency of DSP in the alginate-based dry capsules

\begin{tabular}{|c|c|c|}
\hline \multicolumn{3}{|c|}{ Quantity of DSP per 100 mg of dry capsules, \% } \\
\hline DSP/CB & DSP/CB-HAp & DSP/0 \\
\hline $19.2 \pm 0.2$ & $24.2 \pm 0.3$ & $12.3 \pm 0.1$ \\
\hline
\end{tabular}

The entrapment of drug was almost 2-fold higher in dexamethasone-loaded beads containing CB-derived materials, then in dexamethasone-loaded beads without mineral fillers.

According to the research of Jyothi et al. [30] there are a number of factors that could influence encapsulation efficiency. In this study, preparation conditions of beads were the same for all sample groups, including concentration of alginate solution, ratio of dispersed phase to continuous phase and molecular weight of polymers. Incorporating of $\mathrm{CB}$ or $\mathrm{CB}-\mathrm{HAp}$ microparticles into composition of capsules obviously decreased the ratio of hydrogel-forming polymers (alginate and chitosan) to a mineral filler, and therefore enhanced solidification during stirring in a continuous phase. Drug loss of DSP/0 into continuous phase could therefore occur, because in this case longer time for solidification of capsules in continuous phase was required. DSP concentration $(0.4 \%)$ was taken as a basis, because this concentration of drug is generally established to give a therapeutic effect when prescribed per os or intravenously. For the topical application the DSP concentrations could be dropped to the $0.05 \%-0.1 \%$ range. Though addition of chitosan into continuous phase improves the structure of prepared capsules as was described above, the interaction between DSP and chitosan could possibly contribute to the general low drug encapsulation rate [31]. On the other hand, strong interaction between drug and structural polymer for capsule formation (sodium alginate in this study) is also not recommended, because it could limit drug release into biological medium [32]. To enhance encapsulation efficiency, the increased ratio of dispersed phase to continuous phase could be taken.

FT-IR spectra of dexamethasone-loaded alginate capsules are presented in Fig. 2.

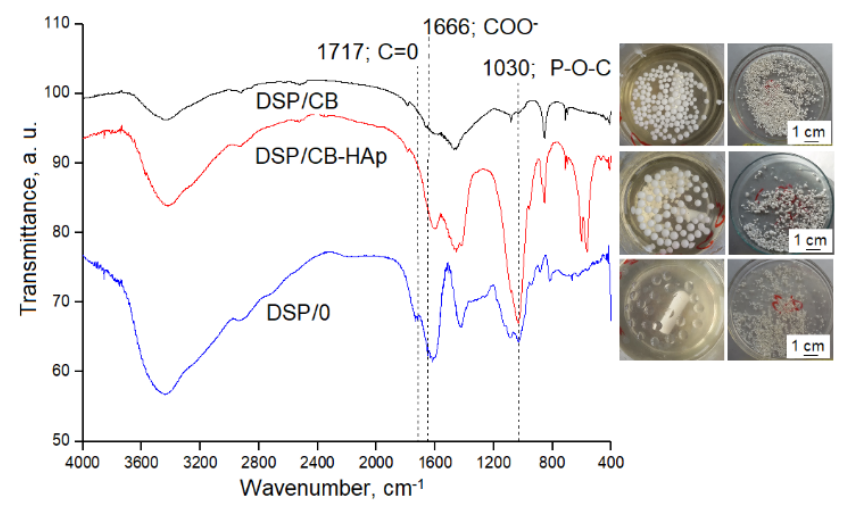

Fig. 2. FT-IR spectra of DSP-loaded alginate capsules: DSP/CB, DSP/CB-HAp and DSP/0. Photographs of capsules in a wet form (left) and lyophilised samples in a dry form (right), are presented along each FT-IR spectrum

In the FT-IR spectra for DSP, the $1717 \mathrm{~cm}^{-1}, 1666 \mathrm{~cm}^{-1}$ and $1030 \mathrm{~cm}^{-1}$ bands of low intensity are visible and originate from the $\mathrm{C}-\mathrm{H}$ stretching vibration of the $\mathrm{C}=\mathrm{O}$, $\mathrm{COO}^{-}$antisymmetric stretching vibration and $-\mathrm{P}-\mathrm{O}-\mathrm{C}$ vibration, respectively [33]. The $-\mathrm{P}-\mathrm{O}-\mathrm{C}$ vibration peak of high intensity is visible in the spectrum of DSP/CB-HAp, because it contains HAp as a mineral filler. Other DSP characteristic absorption peaks are not clearly visible, because of interference with the peaks, generated from functional groups of alginate, CB and chitosan.

The structure and surface morphology of prepared beads were analysed from SEM microphotographs (Fig. 3). 
The mean particle size varied in the range of $0.89-0.93 \pm 0.05 \mathrm{~mm}, \quad 0.91-0.94 \pm 0.08 \mathrm{~mm} \quad$ and $0.98-1.2 \pm 0.04 \mathrm{~mm}$ for DSP/CB, DSP/CB-HAp and $\mathrm{DSP} / 0$, respectively, with a narrow distribution. Although the morphology of all sample groups in wet state was spherical with a diameter of approximately $2 \mathrm{~mm}$, it changed after drying, showing the visible differences among beads with/without mineral fillers.

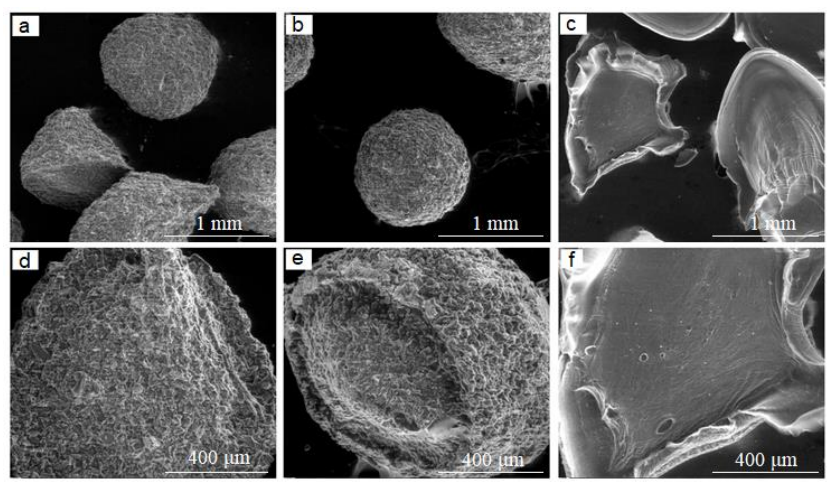

Fig. 3. Microphotographs of dexamethasone-loaded alginate capsules in a dry form: a, d-with $\mathrm{CB}$ microparticles (DSP/CB); b, e-with CB-HAp (DSP/CB-HAp); c, $\mathrm{f}$ - without any mineral filler (DSP/0)

Almost spherical shape of DSP/CB and DSP/CB-HAp remained after lyophilisation. The preservation of spherical shape of DSP/CB and DSP/CB-HAp capsules was also achieved by reinforcing of alginate with mineral fillers. Some folds and cavities were observed, as an evidence of unavoidable contraction force and polymer viscosity increase during any drying process [34]. The rough surface of DSP/CB and DSP/CB-HAp demonstrates the presence and even distribution of mineral fillers. It could be concluded that reinforcing with mineral fillers and lyophilisation as a gradual non-drastic drying process contributed to the preservation of capsules structure. Oppositely, the morphology of DSP/0 changed dramatically, showing number of cracks and wrinkles in the disordered non-spherical structure.

The objective of the swelling behaviour experiment was to simulate the temperature and $\mathrm{pH}$ of biological environment. Normally, human saliva maintains the $\mathrm{pH}$ of oral cavity near neutrality ( $\mathrm{pH}$ in a 6.7-7.3 range) [35], while the $\mathrm{pH}$ of human gingival crevicular fluid is in a $7.5-8.7$ range. For the explanation of the results, it can be assumed that the decrease of $S I$ indicates the disintegration of beads and subsequent release of its load into surrounding medium [36].

The complete disintegration of DSP/CB-HAp beads occurred just after $2 \mathrm{~h}$, indicating rapid release of dexamethasone and CB-HAp from the beads (Fig. 4). In comparison, after $1 \mathrm{~h}$ of soaking in PBS the $S I$ of DSP/CB beads started to decrease, indicating only partial dissolution.

Typical SI for all types of beads was observed at $1 \mathrm{~h}$ data point. After $1 \mathrm{~h}$, an average $S I$ of alginate-based beads without mineral fillers was much higher in comparison to beads with $\mathrm{CB}$ fillers: $121 \pm 34.0,17 \pm 0.8$ and $3 \pm 1.0$ for DSP/0, DSP/CB and DSP/CB-HAp beads, respectively.

$\mathrm{DSP} / 0$ beads had a high $\mathrm{SI}$ due to the hydration of $\mathrm{COO}^{-}$ groups of alginate, as well as $\mathrm{NH}_{3}{ }^{+}$groups of chitosan presented a composition of core matrix. Oppositely, DSP$\mathrm{CB}$ and DSP/CB-HAp samples showed only marginal increase of SI during the experiment because reinforcement of alginate matrix with mineral fillers stabilised the structure of capsules. By taking into account the limited space of periodontal intrabone defects, decreased swelling of capsules for bone repair is greatly desirable.

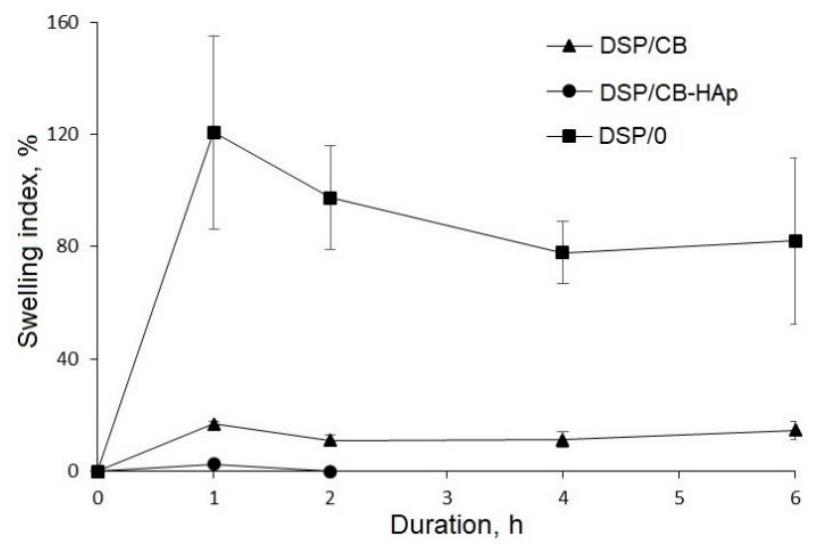

Fig. 4. Swelling behaviour of dexamethasone-loaded alginate beads in PBS at $37^{\circ} \mathrm{C}(\mathrm{pH}=7.2)$

\section{CONCLUSIONS}

Alginate capsules with cuttlebone-derived mineral fillers for bone repair were successfully prepared by ionic cross-linking method. The mean size of dry capsules was in the $0.89-0.94 \pm 0.08 \mathrm{~mm}$ range that is conformable to the size of periodontal intrabone defects. The addition of $5 \%$ glucono-delta-lactone ensured controlled gelation of alginate hydrogel during capsule core formation, also supporting the desirable degradation of poorly soluble calcium compounds. To conclude, an integrated solution for bone repair in endodontic and periodontal therapy was suggested by preparation and characterisation of alginatebased capsules with cuttlebone-derived mineral fillers.

\section{REFERENCES}

1. Younes, I., Rinaudo, M. Chitin and Chitosan Preparation from Marine Sources. Structure, Properties and Applications Marine drugs 13 (3) 2015: pp. 1133-1174. https://doi.org/10.3390/md13031133

2. Cadman, J., Zhou, S., Chen, Y., Li, Q. Cuttlebone: Characterisation, Application and Development of Biomimetic Materials Journal of Bionic Engineering 9 (3) 2012: pp. $367-376$. https://doi.org/10.1016/S1672-6529(11)60132-7

3. Álvarez, A.L, Espinar, F.O., Méndez, J.B. The Application of Microencapsulation Techniques in the Treatment of Endodontic and Periodontal Diseases Pharmaceutics 3 (3) 2011: pp. $538-571$. https://doi.org/10.3390/pharmaceutics3030538

4. Harini, G., Kaarthikeyan, G. Advanced Drug Delivery Systems in Treating Periodontal Diseases - A Review Journal of Dental and Medical Sciences 13(1) 2014: pp. 27 - 32.

5. Gauthier, O., Bouler, J., Weiss, P., Bosco, J., Daculsi, G., Aguado, E. Kinetic Study of Bone Ingrowth and Ceramic Resorption Associated with the Implantation of Different Injectable Calcium-Phosphate Bone Substitutes Journal of Biomedical Materials Research Part A 47 (1) 1999: pp. $28-35$.

https://doi.org/10.1002/(SICI)1097-

4636(199910)47:1<28::AID-JBM4>3.0.CO;2-P 
6. Nair, S.C., Anoop, K.R. Intraperiodontal Pocket: An Ideal Route for Local Antimicrobial Drug Delivery Journal of Advanced Pharmaceutical Technology and Research 3 (1) 2012: pp. $9-15$.

https://doi.org/10.4103/2231-4040.93558

7. Gupta, G. Clinical and Radiographic Evaluation of Intrabony Defects in Localized Aggressive Periodontitis Patients with Platelet Rich Plasma/Hydroxyapatite Graft: A Comparative Controlled Clinical Trial Contemporary Clinical Dentistry 5 (4) 2014: pp. 445-451. https://doi.org/10.4103/0976-237X.142806

8. Florek, M., Fornal, E., Gómez-Romero, P., Zieba, E., Paszkowicz, W., Lekki, J., Nowak, J., Kuczumow, A. Complementary Microstructural and Chemical Analyses of Sepia Officinalis Endoskeleton Materials Science and Engineering: $C 29$ (4) 2009: pp. 1220-1226. https://doi.org/10.1016/j.msec.2008.09.040

9. Rocha, J., Lemos, A., Agathopoulos, S., Valério, P., Kannan, S., Oktar, F., Ferreira, J.M.F. Scaffolds for Bone Restoration from Cuttlefish Bone 37 (6) 2005: pp. $850-857$. https://doi.org/10.1016/j.bone.2005.06.018

10. Yukna, R.A. Synthetic Bone Grafts in Periodontics Periodontology 20001 (1) 1993: pp. $92-99$. https://doi.org/10.1111/j.1600-0757.1993.tb00210.x

11. Soares, S., Silva, A., Correia, I. Porous Bioglass Scaffold for Orthopedics Applications Materials Science (Medžiagotyra) 22 (2) 2016: pp. 279-284.

http://dx.doi.org/10.5755/j01.ms.22.2.8581

12. Lee, K.Y., Mooney, D.J. Alginate: Properties and Biomedical Applications Progress in Polymer Science 37 (1) 2012: pp. $106-126$.

https://doi.org/10.1016/j.progpolymsci.2011.06.003

13. Tønnesen, H.H., Karlsen, J. Alginate in Drug Delivery Systems Drug Development and Industrial Pharmacy 28 (6) 2002: pp. $621-630$. https://doi.org/10.1081/DDC-120003853

14. Govender, S., Lutchman, D., Pillay, V., Chetty, D., Govender, T. Enhancing Drug Incorporation into Tetracycline-loaded Chitosan Microspheres for Periodontal Therapy Journal of Microencapsulation 23 (7) 2006: pp. $750-761$. https://doi.org/10.1080/02652040600789229

15. Renvert, S., Lessem, J., Dahlén, G., Lindahl, C., Svensson, M. Topical Minocycline Microspheres Versus Topical Chlorhexidine Gel as an Adjunct to Mechanical Debridement of Incipient Peri-implant Infections: a Randomized Clinical Trial Journal of Clinical Periodontology 33 (5) 2006: pp. 362-369. 10.1111/j.1600-051X.2006.00919.x

16. Jain, N., Jain, G.K., Javed, S., Iqbal, Z., Talegaonkar, S., Ahmad, F.J., Khar, R.K. Recent Approaches for the Treatment of Periodontitis Drug Discovery Today 13 (21) 2008: pp. $932-943$. https://doi.org/10.1016/j.drudis.2008.07.010

17. Patel, P., Mundargi, R.C., Babu, V.R., Jain, D., Rangaswamy, V., Aminabhavi, T.M. Microencapsulation of Doxycycline into Poly (lactide-co-glycolide) by Spray Drying Technique: Effect of Polymer Molecular Weight on Process Parameters Journal of Applied Polymer Science 108 (6) 2008: pp. $4038-4046$. https://doi.org/10.1002/app.28040

18. Ferraz, M., Mateus, A., Sousa, J., Monteiro, F. Nanohydroxyapatite Microspheres as Delivery System for Antibiotics: Release Kinetics, Antimicrobial Activity, and Interaction with Osteoblasts Journal of biomedical Materials Research Part A 81 (4) 2007: pp. 994-1004.

\section{https://doi.org/10.1002/jbm.a.31151}

19. Stanislavov, A., Sukhodub, L.B., Yanovska, A.A., Kuznetzov, V.N., Sukhodub, L.F. Alginate-hydroxyapatite Beads for Medical Application Proceedings of the International Conference Nanomaterials: Applications and Properties 3 (2) 2014: pp. 1-2.

20. Ivankovic, H., Tkalcec, E., Orlic, S., Ferrer, G.G., Schaulperl, Z. Hydroxyapatite Formation from Cuttlefish Bones: Kinetics Journal of Materials Science: Materials in Medicine 21 (10) 2010: pp. 2711-2722. https://doi.org/10.1007/s10856-010-4115-4

21. Pasparakis, G., Bouropoulos, N. Swelling Studies and in Vitro Release of Verapamil from Calcium Alginate and Calcium Alginate-Chitosan beads International Journal of Pharmaceutics 323 (1) 2006: pp. 34-42. https://doi.org/10.1016/j.ijpharm.2006.05.054

22. Sun, J., Tan, H. Alginate-based Biomaterials for Regenerative Medicine Applications Materials 6(4) 2013: pp. $1285-1309$. https://doi.org/10.3390/ma6041285

23. Rehm, B.H. Alginates: Biology and Applications. Springer, Berlin, 2009: pp. $175-210$. https://doi.org/10.1007/978-3-540-92679-5

24. Sariibrahimoglu, K. Strategies to Accelerate the Degradation of Injectable Calcium Phosphate-based Composite Materials for Bone Regeneration. 'sHertogenbosch: Uitgeverij BOXPress; 2014.

25. Nelson, D., Featherstone, J., Duncan, J., Cutress, T. Paracrystalline Disorder of Biological and Synthetic Carbonate-substituted Apatites Journal of Dental Research 61 (11) 1982: pp. $1274-1281$. $10.1177 / 00220345820610111301$

26. Ramsey, L.B., Pounds, S., Cheng, C., Cao, X., Yang, W., Smith, C., Karol, S.E., Liu, C., Panetta, J.C., Inaba, H., Rubnitz, J.E., $\quad$ Metzger, M.L., $\quad$ Ribeiro, R.C., Sandlund, J.T., Jeha, S., $\quad$ Pui, C.H., $\quad$ Evans, W.E., Relling, M.V. Genetics of Pleiotropic Effects of Dexamethasone Pharmacogenet Genomics 27 (8) 2017: pp. 294-302. https://doi.org/10.1097/FPC.0000000000000293

27. McCullers, J.A. Preventing and Treating Secondary Bacterial Infections with Antiviral Agents Antiviral Therapy 16 (2) 2011: pp. 123-135. https://doi.org/10.3851/IMP1730

28. Obradović, N.S., $\quad$ Krunić, T.Z., $\quad$ Trifković, K.T., Bulatović, M.L., Rakin, M.P., Rakin, M.B., Branko, M., Bugarski, B.M. Influence of Chitosan Coating on Mechanical Stability of Biopolymer Carriers with Probiotic Starter Culture in Fermented Whey Beverages International Journal of Polymer Science 2015 2015: pp. 1-8. http://dx.doi.org/10.1155/2015/732858

29. Yang, Y., Campanella, O.H., Hamaker, B.R., Zhang, G., Gu, Z. Rheological Investigation of Alginate Chain Interactions Induced by Concentrating Calcium Cations Food Hydrocolloids 30 (1) 2013: pp. 26-32. https://doi.org/10.1016/j.foodhyd.2012.04.006

30. Jyothi, N.V.N., $\quad$ Prasanna, P.M., $\quad$ Sakarkar, S.N., Prabha, K.S., Ramaiah, P.S., $\quad$ Srawan, G. Microencapsulation Techniques, Factors Influencing Encapsulation Efficiency Journal of Microencapsulation 27 (3) 2010: pp. $187-197$. https://doi.org/10.3109/02652040903131301

31. Doustgani, A., Farahani, E.V., Imani, M., Doulabi, A.H. Dexamethasone Sodium Phosphate Release from Chitosan Nanoparticles Prepared by Ionic Gelation Method Journal of Colloid Science and Biotechnology 1 (1) 2012: pp. $42-50$. 
https://doi.org/10.1166/jcsb.2012.1009

32. Crotts, G., Park, T.G. Stability and Release of Bovine Serum Albumin Encapsulated within Poly (D, L-lactide-coglycolide) Microparticles Journal of Controlled Release 44 (2) 1997: pp. $123-134$.

https://doi.org/10.1016/S0168-3659(96)01511-8

33. Tang, R., Guo, X., Ji, D., Kan, J. Synthesis and Characterization of Functional Superparamagnetic Nanocomposites Containing $\quad \gamma$-Fe2 O3, Dexamethasone Sodium Phosphate and Polyaniline Indian Journal of Engineering and Materials Science 21 (4) 2014: pp. $207-213$.

34. Kurmen, C., Elena, J., Gómez Alvarez, M.I, Villamizar Rivero, L.F. Microencapsulation of a Colombian
Spodoptera Frugiperda Nucleopolyhedrovirus with Eudragit ${ }^{\circledR}$ S100 by Spray Drying Brazilian Archives of Biology and Technology 58 (3) 2015: pp. 468-476. http://dx.doi.org/10.1590/S1516-8913201500453

35. Baliga, S., Muglikar, S., Kale, R. Salivary pH: A Diagnostic Biomarker Journal of Indian Society of Periodontology 17 (4) 2013: pp. $461-465$.

https://doi.org/10.4103/0972-124X.118317

36. Cook, M.T., Tzortzis, G., Charalampopoulos, D., Khutoryanskiy, V.V. Production and Evaluation of Dry Alginate-Chitosan Microcapsules as an Enteric Delivery Vehicle for Probiotic Bacteria Biomacromolecules 12 (7) 2011: pp. 2834-2840.

https://doi.org/10.1021/bm200576h 